\title{
ON THE MAXIMUM OF PERIODIC INTEGER-VALUED SEQUENCES WITH EXPONENTIAL TYPE TAILS VIA MAX-SEMISTABLE LAWS
}

\author{
A. HALL AND M. G. TEMIDO
}

\begin{abstract}
In this work we study the limiting distribution of the maximum term of periodic integer-valued sequences with marginal distribution belonging to a particular class where the tail decays exponentially. This class does not belong to the domain of attraction of any max-stable distribution. Nevertheless, we prove that the limiting distribution is max-semistable when we consider the maximum of the first $k_{n}$ observations, for a suitable sequence $\left\{k_{n}\right\}$ increasing to infinity. We obtain an expression for calculating the extremal index of sequences satisfying certain local conditions similar to conditions $D^{(m)}\left(u_{n}\right), m \in \mathbb{N}$, defined by Chernick et al. (1991). We apply the results to a class of max-autoregressive sequences and a class of moving average models. The results generalize the ones obtained for the stationary case.
\end{abstract}

Keywords: integer-valued periodic sequences, max-semistable laws, binomial thinning.

\section{Introduction}

Integer-valued and in particular count data arise in a variety of contexts and the need to model this type of data adequately has given rise to a considerable diversity of models. For an overview of the literature in this area refer to the review work by McKenzie (2003) and the monographs of MacDonald and Zucchini (1997) or Kedem and Fokianos (2002). Count data models may be divided into two broad categories: observation-driven and parameter driven models. Whereas the latter

Received November 7, 2007.

This work was supported by Fundação para a Ciência e a Tecnologia (Portugal) through Unidade de Investigação Matemática e Aplicações of University of Aveiro and Center for Mathematics of University of Coimbra. 
rely on a latent process connecting the observations, the former specify a direct link between current and past observations. In this paper we consider several classes of observation-driven models. Some of these models are obtained replacing multiplication in conventional models by binomial thinning defined as follows,

$$
\beta \circ Z=\sum_{s=1}^{Z} \beta_{s}(\beta), \beta \in[0,1],
$$

where $\left\{B_{s}(\beta)\right\}$ is an independent and identically distributed (i.i.d.) sequence of Bernoulli r.v.'s satisfying $P\left[B_{s}(\beta)=1\right]=\beta$ and $Z$ is a nonnegative integer-valued random variable (r.v.). Models based on thinning were first considered by McKenzie (1985) and have been elaborated on in subsequent papers by McKenzie $(1986,1988)$, Al-Osh and Alzaid (1987, 1988), Alzaid and Al-Osh (1988, 1990, 1993), Du and Li (1991), and Brännäs and Hall (2001) among other authors.

When the extremes of discrete time series are of interest several drawbacks emerge since many integer-valued distributions do not belong to the domain of attraction of any extreme value distribution. Examples include the Binomial, Negative Binomial and Poisson distributions. Nevertheless, there has been some interest in this topic and several authors have already studied the extremal behavior of several integer-valued time series models. Anderson (1970) gave a noticeable contribution to the study of the extremal properties of integer-valued i.i.d. sequences and, as an example of application, the author analyzed the behavior of the maximum queue length for $M / M / 1$ queues. McCormick and Park (1992) were the first to study the extremal properties of some models obtained as discrete analogues of continuous models, replacing scalar multiplication by random thinning. More recently, Hall (2001) provided results regarding the limiting distribution of the maximum of sequences within a generalized class of integer-valued moving averages driven by i.i.d. 
heavy-tailed innovations. Extensions for exponential type-tails innovations have been studied by Hall (2003). Also of interest for the present work is the paper by Hall (1996) who studies the extremes of a maxautoregressive model for integer-valued data.

Most of the work on extremes of integer-valued models consider stationary models and as far as we know only one recent work considers the extremes of non-stationary sequences: Hall and Scotto (2006) who consider the extremes of periodic integer-valued models with exponential type tails.

Hall and Scotto (2006) consider periodic integer-valued sequences with exponential type tails, but using a different approach. In all cases considered, the marginal distribution of the process and the distribution of the innovations do not belong to the domain of attraction of any extreme value distribution but are included in a class of distributions considered by Anderson (1970) for which the maxima in an i.i.d. setting possesses a quasi-stable limiting behavior. Anderson proved that an integer-valued distribution function (d.f.) $F$, with infinite right endpoint, satisfies

$$
\left.\lim _{n \rightarrow+\infty} \frac{1-F(n-1)}{1-F(n)}=r, \text { with } r \text { in }\right] 1,+\infty[,
$$

if and only if

$$
\left\{\begin{array}{l}
\limsup _{n \rightarrow \infty} F^{n}\left(x+b_{n}\right) \leq \exp \left(-r^{-x}\right) \\
\liminf \inf _{n \rightarrow \infty} F^{n}\left(x+b_{n}\right) \geq \exp \left(-r^{-(x-1)}\right)
\end{array}\right.
$$

for any real $x$ and $b_{n}$ conveniently chosen. We shall say that a distribution belongs to Anderson's class $\left(F \in D_{r}\right.$ (Anderson $\left.)\right)$ if it satisfies (1).

In an attempt to overcome the presence of limiting bounds instead of a well defined limiting distribution, Temido (2000) proved that (1) is necessary and sufficient for the existence of a nondecreasing positive integer sequence $\left\{k_{n}\right\}$ satisfying

$$
\left.\lim _{n \rightarrow+\infty} \frac{k_{n+1}}{k_{n}}=r, \quad r \in\right] 1,+\infty[,
$$


and of a real sequence $\left\{u_{n}\right\}$ such that $k_{n}\left(1-F\left(u_{n}\right)\right) \rightarrow \tau>0$, as $n \rightarrow \infty$ for some $\tau>0$. So, if instead of looking at the maximum term of the first $n$ observations we look at the maximum term of the first $k_{n}$ observations, where $\left\{k_{n}\right\}$ satisfies (2), we can obtain a well defined limiting distribution for the maximum term. The limiting distribution belongs to a class of distributions known as max-semistable (MSS). In Section 2.1 we provide a brief description of the basic theoretical results related to the MSS class.

Sections 2.2 and 2.3 provide preliminary results concerning the extremes of stationary sequences with margins in the domain of attraction of a MSS distribution, and the extremes of periodic sequences.

Section 3 provides the general results which allow us to obtain the limiting distribution of periodic sequences with margins in the domain of attraction of a MSS distribution.

In section 4 we obtain the limiting distribution of the maximum for three classes of periodic integer-valued models. The first class is an infinite moving average model obtained as a discrete analogue of the classical MA models, replacing multiplication by binomial thinning. For this model the maximum term behaves as if the sequence was independent. The second class is a first order max-autoregressive periodic sequence for which clusters of exceedances are formed and therefore the extremal index is less than unity. Both these classes have been considered by Hall and Scotto (2006) although in this work we use a different approach. Whereas Hall and Scotto only obtain bounds for the limiting distribution of the maximum we manage to obtain a non-degenerate limiting distribution for the maximum under the MSS setup. Finally, we consider a second order max-autoregressive periodic sequence which also has clusters of exceedances but with different characteristics. Again, the extremal index is less than unity.

\section{Preliminary results}


2.1. Max-semistable laws. The class of max-stable laws, also known as extreme value distributions, arises as the possible limiting distributions for the linearized maxima of $n$ i.i.d. r.v.'s, as $n \rightarrow \infty$. If instead of considering $n$ r.v.'s we consider $k_{n}$ r.v.'s, where $\left\{k_{n}\right\}$ is a nondecreasing positive integer-valued sequence satisfying

$$
\lim _{n \rightarrow+\infty} \frac{k_{n+1}}{k_{n}}=r, \quad r \in[1,+\infty[,
$$

then we obtain a larger class of possible limiting distributions known as the MSS distributions. The class of MSS distributions was introduced by Pancheva (1992) and Grinevich (1992). This class includes nondegenerate limiting distributions for the maxima of i.i.d. r.v.'s with either discrete distributions or multi-modal continuous distributions which are not extreme value distributions.

Following Grinevich (1992) we will say that a d.f. $G$ on $\mathbb{R}$ is MSS if there are reals $r>1, \gamma>0$ and $\beta$ such that

$$
G(x)=G^{r}(x / \gamma+\beta), x \in \mathbb{R},
$$

or equivalently, if there is a sequence of i.i.d. r.v.'s with d.f. $F$ and two real sequences $\left\{a_{n}>0\right\}$ and $\left\{b_{n}\right\}$ for which

$$
\lim _{n \rightarrow+\infty} F^{k_{n}}\left(x / a_{n}+b_{n}\right)=G(x),
$$

for each continuity point of $\mathrm{G}$, with $\left\{k_{n}\right\}$ satisfying (3).

Analytically, the MSS laws can be written as follows.

Theorem 2.1 (Grinevich, 1992). (1) For $\gamma>0$ and $\beta=t(1-1 / \gamma)$ with $t \in \mathbb{R}$ the solutions of (4) are the following distributions:

$$
\begin{aligned}
& \left.G_{1}(x)=\exp \left\{-(t-x)^{\alpha} \nu(\ln (t-x))\right\}, x \in\right]-\infty, t[, \\
& \left.G_{2}(x)=\exp \left\{-(x-t)^{-\alpha} \nu(\ln (x-t))\right\}, x \in\right] t,+\infty[,
\end{aligned}
$$

where $\alpha=|\log r / \log \gamma|$ and $\nu$ is a periodic positive and bounded function with period $p=|\log \gamma|$. 
(2) For $\gamma=1$ and $\beta>0$ the solutions of (4) are of the form $G_{3}(x)=$ $\exp \left\{e^{-\alpha x} \nu(x)\right\}, x \in \mathbb{R}$, where $\alpha=\ln r / \beta$, and $\nu$ is a periodic positive and bounded function with period $\beta$.

The general definition of the MSS laws is given in Grinevich (1992, 1993). Canto e Castro et al. (2000) give a characterization of the MSS laws simpler than the one given by Grinevich (1993). Necessary and sufficient conditions on $F$ such that (4) holds are also given in Canto e Castro et al. (2000).

If limit (5) holds we shall say that $F$ belongs to the domain of attraction of $G$.

Considering now discrete r.v.'s, Temido (2000) proved the following theorem.

Theorem 2.2 (Temido, 2000). For any discrete d.f. $F$ defined over the set $\left\{x_{n}\right\}$ and such that $\lim _{x \rightarrow w_{F}^{-}} F(x)=1$, a necessary and sufficient condition for the existence of a nondecreasing positive integer sequence $\left\{k_{n}\right\}$ satisfying (3) and of a real sequence $\left\{u_{n}\right\}$ such that $F^{k_{n}}\left(u_{n}\right)$ has a non-degenerate limit is given by

$$
\lim _{n \rightarrow+\infty} \frac{1-F\left(x_{n-1}\right)}{1-F\left(x_{n}\right)}=r .
$$

This theorem allows us to prove the previously mentioned result by which any d.f. in Anderson's class, satisfying (1), can only belong to the domain of attraction of a MSS distribution. Furthermore, the limit lies in the discrete Gumbel family, included in $G_{3}$ as stated in the following theorem.

Theorem 2.3 (Temido, 2002). Let $F$ be an integer-valued d.f. with infinite right endpoint. If there is a nondecreasing integer-valued and positive sequence $\left\{k_{n}\right\}$ satisfying (2) and two real sequences $\left\{a_{n}>0\right\}$, and $\left\{b_{n}\right\}$ such that $\lim _{n \rightarrow+\infty} F^{k_{n}}\left(x / a_{n}+b_{n}\right)=G(x)$, then $G$ must is of form

$$
G(x)=\exp \left(-r^{-[x]}\right), \quad x \in \mathbb{R},
$$


if and only if $\lim _{n \rightarrow+\infty} \frac{1-F(n)}{1-F(n+1)}=r>1$.

As stated before, unlike any extreme value distribution a MSS distribution may have several modes or may be discrete. Examples of d.f.'s which do not belong to the domain of attraction of any extreme value distribution but belong to the domain of attraction of a max-semistable law are the Negative Binomial, $N B(n, p)$, and von Misès distribution given by $F(x)=1-\exp (-x-\operatorname{sen} x)$, for $x \geq 0$. They belong to the domain of attraction of $G(x)=\exp \left(-p^{[x]}\right)$ and $G(x)=\exp (-\exp (-x-\operatorname{sen} x))$, with real $x$, respectively.

Note that since there are infinitely sequences $\left\{k_{n}\right\}$ satisfying (3), the choice of the normalizing constants $a_{n}$ and $b_{n}$ is wider than in the classic extreme-value setup. This flexibility may sometimes make the proofs neater but in many circumstances it adds extra complexity to them.

2.2. Stationary sequences and max-stability. Temido and Canto e Castro (2003) consider all stationary sequences, $\left\{X_{n}\right\}$, satisfying a new dependence restriction, $D_{k_{n}}\left(u_{n}\right)$, which generalizes Leadbetter's condition $D\left(u_{n}\right)$ (Leadbetter et al. (1983)). They prove that when the sequence $\left\{X_{n}\right\}$ satisfies $D_{k_{n}}\left(u_{n}\right)$ the limiting behavior of the maximum term $M_{k_{n}}=\max \left\{X_{1}, \ldots, X_{k_{n}}\right\}$ can be inferred from the limiting behavior of the maximum, $\hat{M}_{k_{n}}$, of the associated independent sequence, $\left\{\hat{X}_{n}\right\}$, using an extension of the well known extremal index. Thus, the corresponding classical result of Gnedenko and Leadbetter's Extremal Types Theorem are generalized.

Definition 2.1 (Temido and Canto e Castro, 2003). Let $\left\{k_{n}\right\}$ be a nondecreasing sequence of positive integers. The sequence of r.v.'s $\left\{X_{n}\right\}$ satisfies condition $D_{k_{n}}\left(u_{n}\right)$ if for any integers $1 \leq i_{1}<\ldots<i_{p}<j_{1}<$ 
$\ldots<j_{q} \leq k_{n}$, for which $j_{1}-i_{p}>\ell_{n}$, we have

$$
\begin{aligned}
& \mid P\left(X_{i_{1}} \leq u_{n}, \ldots, X_{i_{p}} \leq u_{n}, X_{j_{1}} \leq u_{n}, \ldots, X_{j_{q}} \leq u_{n}\right)- \\
& \quad-P\left(X_{i_{1}} \leq u_{n}, \ldots, X_{i_{p}} \leq u_{n}\right) P\left(X_{j_{1}} \leq u_{n}, \ldots, X_{j_{q}} \leq u_{n}\right) \mid \leq \alpha_{n, \ell_{n}}
\end{aligned}
$$

where $\lim _{n \rightarrow+\infty} \alpha_{n, \ell_{n}}=0$ for some sequence $\ell_{n}=o_{n}\left(k_{n}\right)$.

Theorem 2.4 (Temido and Canto e Castro, 2003). Let $\left\{k_{n}\right\}$ be a nondecreasing positive integer sequence satisfying (3). Let $\left\{X_{n}\right\}$ be a stationary sequence and let $\left\{a_{n}>0\right\}$ and $\left\{b_{n}\right\}$ be real sequences such that $\limsup k_{n}\left(1-F\left(x / a_{n}+b_{n}\right)\right)<+\infty$ and $P\left(a_{n}\left(M_{k_{n}}-b_{n}\right) \leq x\right)$ converges $n \rightarrow+\infty$ to $G(x)$ for each continuity point of the nondegenerate d.f. G. If $\left\{X_{n}\right\}$ satisfies $D_{k_{n}}\left(x / a_{n}+b_{n}\right)$, for all $x$ in $\mathbb{R}$, then $G$ belongs to one of the three classes of max-semistable laws.

2.3. Extremes of periodic sequences. In this work we say that $\left\{X_{n}\right\}$ is T-periodic with period $T$ if there exists an integer $T \geq 1$ such that, for each choice of integers $1 \leq i_{1}<i_{2}<\cdots<i_{n},\left(X_{i_{1}}, \ldots, X_{i_{n}}\right)$ and $\left(X_{i_{1}+T}, \ldots, X_{i_{n}+T}\right)$ are identically distributed. The period $T$ will be considered the smallest integer satisfying the above definition.

Alpuim (1988) has studied the extremes of T-periodic sequences of independent r.v.'s. Attending that, for $\bar{F}(x)=\frac{1}{T} \sum_{i=1}^{T} F_{i}(x)$,

$$
n\left(1-\bar{F}\left(u_{n}\right)\right) \rightarrow \tau \text { iif } P\left(M_{n} \leq u_{n}\right) \sim\left(\prod_{i=1}^{T} F_{i}\left(u_{n}\right)\right)^{\frac{n}{T}} \rightarrow e^{-\tau},
$$

where $\left\{u_{n}\right\}$ is a sequence of real constants and $M_{n}$ denotes the maximum of the first $n$ observations, she proved that the class of possible limit distributions for $M_{n}$, under linear normalization, coincides with the class MS. In addition, for T-periodic dependent sequences satisfying Leadbetter's $D\left(u_{n}\right)$ condition, Alpuim generalized Leadbetter's extremal types theorem and the notion of extremal index. 
The study of the extremal properties of stationary sequences is frequently based on the verification of appropriate dependence conditions as for instance Leadbetter's $D\left(u_{n}\right)$ condition and condition $D^{(m)}\left(u_{n}\right), m \in$ $\mathbb{N}$, defined by Chernick et al. (1991). Ferreira and Martins (2003) have defined analogous conditions for periodic sequences and as in Chernick et al. they provide an expression to determine the extremal index of the sequence.

Definition 2.2 (Ferreira and Martins, 2003). Let $m \geq 1$ be a fixed integer and $\left\{X_{n}\right\}$ a T-periodic sequence satisfying $D\left(u_{n}\right)$ with mixing coefficient $\alpha_{n, \ell_{n}}$. The condition $D_{T}^{(m)}\left(u_{n}\right)$ holds for $\left\{X_{n}\right\}$ when there exists a sequence of integers $\left\{s_{n}\right\}_{n \in \mathbb{N}}$ such that

$$
\lim _{n \rightarrow \infty} s_{n}=+\infty, \lim _{n \rightarrow \infty} s_{n} \frac{\ell_{n}}{n}=0, \lim _{n \rightarrow \infty} s_{n} \alpha_{n, \ell_{n}}=0 \text { and } \lim _{n \rightarrow \infty} S_{\left[\frac{n}{s_{n} T}\right]}^{(m)}=0
$$

where

$$
S_{\left[\frac{n}{s_{n} T}\right]}^{(1)}=\frac{n}{T} \sum_{i=1}^{T} \sum_{j=i+m}^{\left[\frac{n}{s_{n} T}\right] T} P\left(X_{i}>u_{n}, X_{j}>u_{n}\right),
$$

and for $m \geq 2$

$$
S_{\left[\frac{n}{s_{n} T}\right]}^{(m)}=\frac{n}{T} \sum_{i=1}^{T} \sum_{j=i+m}^{\left[\frac{n}{s_{n} T}\right] T} P\left(X_{i}>u_{n}, X_{j-1} \leq u_{n}<X_{j}\right) .
$$

When $D\left(u_{n}\right)$ and $D_{T}^{(m)}\left(u_{n}\right)$ hold for a particular sequence the limiting distribution of the maximum term and hence the extremal index may be derived. Ferreira and Martins (2003) provide an expression for the extremal index:

$$
\theta=\lim n \frac{n \frac{1}{T} \sum_{i=1}^{T}\left(X_{i}>u_{n} \geq M_{i+1, i+m-1}\right)}{n \frac{1}{T} \sum_{i=1}^{T} P\left(X_{i}>u_{n}\right)},
$$

where $M_{i, j}=\max _{i \leq l \leq j}\left\{X_{l}\right\}$ and $M_{i, j}=-\infty$ if $i>j$. 
Hall and Scotto (2006) have generalized the procedure for periodic sequences with marginal distributions in Anderson's class. In this case the limiting distribution of the maximum term is bounded between two Gumbel type distributions.

Theorem 2.5 (Hall and Scotto (2006)). Suppose that for $m \geq 1$ the conditions $D\left(u_{n}\right)$ and $D_{T}^{(m)}\left(u_{n}\right)$ hold for the T-periodic integer-valued sequence $\left\{X_{n}\right\}$, with $F_{s} \in D_{r_{s}}$ (Anderson), for $s=1, \ldots, T$, where $\left\{u_{n}\right\}_{n \in \mathbb{N}}$ is a sequence of the form $u_{n}=x+b_{n}$. Then, there exists a value $0 \leq \theta \leq 1$ such that

$$
\left\{\begin{array}{l}
\limsup _{n \rightarrow \infty} P\left(M_{n} \leq x+b_{n}\right) \leq e^{-\theta \frac{1}{T} \sum_{s=1}^{T} r_{s}^{-x}} \\
\liminf _{n \rightarrow \infty} P\left(M_{n} \leq x+b_{n}\right) \geq e^{-\theta \frac{1}{T} \sum_{s=1}^{T} r_{s}^{-(x-1)}}
\end{array}\right.
$$

if and only if

$$
\frac{\frac{n}{T} \sum_{s=1}^{T} P\left(X_{s}>u_{n}>M_{s+1, s+m-1}\right)}{\frac{n}{T} \sum_{s=1}^{T} P\left(X_{s}>u_{n}\right)} \rightarrow \theta, n \rightarrow \infty .
$$

The parameter $\theta$ is referred to as the extremal index due to its similarity with the conventional extremal index.

\section{General results}

In this section we will obtain a counterpart for Theorem 2.5 using the MSS distributions. We will be able to obtain a well-defined limiting distribution for the maximum term of periodic integer-valued sequences, instead of bounds as in Theorem 2.5.

For simplicity, if $E$ is any set of integers we will denote $\max \left\{X_{j}, j \in\right.$ $E$ \} by $M(E)$ and we will say that $E$ is an interval if the integers are consecutive. Moreover if $E=\left\{j_{1}, \ldots, j_{2}\right\}$ we will say that it has length $j_{2}-j_{1}+1$ and we will also say that it is separated by $i_{1}-j_{2}$ from $F=\left\{i_{1}, \ldots, i_{2}\right\}, i_{1}>j_{2}$.

First we recall how the condition $D_{k_{n}}\left(u_{n}\right)$ gives an asymptotic independence for maxima on separated sets, which is appropriate for the 
subsequent results. We must remark that neither stationarity nor Tperiodicity are considered assumptions. Indeed, if there is a sequence $\left\{k_{n}\right\}$ of positive integers and a real sequence $\left\{u_{n}\right\}$ such that $\left\{X_{n}\right\}$ satisfies $D_{k_{n}}\left(u_{n}\right)$, then, for any subintervals of $\left\{1,2, \ldots, k_{n}\right\}, E_{1}, E_{2}, \ldots, E_{t_{n}}$, such that any two of them are separated by $\ell_{n}$ integers, we have

$$
\left|P\left(\bigcap_{j=1}^{t_{n}}\left\{M\left(E_{j}\right) \leq u_{n}\right\}\right)-\prod_{j=1}^{t_{n}} P\left(M\left(E_{j}\right) \leq u_{n}\right)\right| \leq\left(t_{n}-1\right) \alpha_{n, \ell_{n}} .
$$

Let $\left\{s_{n}\right\}$ be an integer-valued sequence satisfying

$$
\lim _{n \rightarrow+\infty} s_{n}=+\infty, \quad \lim _{n \rightarrow+\infty} s_{n} \alpha_{n, \ell_{n}}=0 \text { and } \lim _{n \rightarrow+\infty} \frac{\ell_{n} s_{n}}{k_{n}}=0 .
$$

In what follows we consider, without loss of generality, that $\ell_{n}$ is a multiple of $T$. In fact, if it is not, we can consider $\ell_{n}^{*}=\left[\frac{\ell_{n}}{T}\right] T$. For any integer $m \geq 0$, let $r_{n, m}=\left[\frac{k_{n-m}}{r^{-m} s_{n} T}\right]$ and $\beta_{n, m}$ be integers such that

$$
\left(\beta_{n, m}-1\right) r_{n, m} T \leq k_{n-m} \leq \beta_{n, m} r_{n, m} T \text {. }
$$

Under these assumptions we can obtain the following limits:

$$
\begin{array}{ll}
\text { i) } \lim _{n \rightarrow+\infty} \frac{\beta_{n, m} \ell_{n}}{k_{n-m}}=0 & \text { ii) } \lim _{n \rightarrow+\infty} \frac{\beta_{n, m} r_{n, m} T}{k_{n-m}}=1 \\
\text { iii) } \lim _{n \rightarrow+\infty} \beta_{n, m} \alpha_{n, \ell_{k_{n}}}=0 & \text { iv) } \lim _{n \rightarrow+\infty} \frac{\beta_{n, m}}{r^{-m} s_{n}}=1 \\
\text { v) } \lim _{n \rightarrow+\infty} \frac{r_{n, 0}}{r_{n, m}}=1 & \text { vi) } \lim _{n \rightarrow+\infty} \frac{\beta_{n, 0}}{\beta_{n, m}}=r^{m} .
\end{array}
$$

Let $m \geq 0$ be a fixed integer and, for large $n$, divide the first $\beta_{n, m} r_{n, m} T$ integers into $2 \beta_{n, m}$ consecutive intervals as follows

$$
\begin{aligned}
& I_{1}=\left\{1, \ldots, r_{n, m} T-\ell_{n}\right\}, \quad I_{1}^{*}=\left\{r_{n, m} T-\ell_{n}+1, \ldots, r_{n, m} T\right\} \\
& I_{\beta_{n, m}}=\left\{\ldots, \beta_{n, m} r_{n, m} T-\ell_{n}\right\}, I_{\beta_{n, m}}^{*}=\left\{\beta_{n, m} r_{n, m} T-\ell_{n}+1, \ldots, \beta_{n, m} r_{n, m} T\right\}
\end{aligned}
$$


where $I_{1}, \ldots, I_{\beta_{n, m}}$ are separated by $\ell_{n}$ and have all the same length equal to $r_{n, m} T-\ell_{n}$.

The following result is the expected generalization of Lemma 4 in Temido and Canto e Castro (2003).

Lemma 3.1. Let $\left\{k_{n}\right\}$ be an integer sequence satisfying (3) and let $\left\{u_{n}\right\}$ be a real sequence such that $\lim \sup k_{n}\left(1-\bar{F}\left(u_{n}\right)\right)<+\infty$. Suppose that the T-periodic sequence $\left\{X_{n}\right\}^{n \rightarrow+\infty}$ satisfies $D_{k_{n}}\left(u_{n}\right)$. Hence, for all integers $m \geq 0$,

$$
\begin{aligned}
& \lim _{n \rightarrow+\infty}\left\{P\left(M_{k_{n-m}} \leq u_{n}\right)-P^{\beta_{n, m}}\left(M_{r_{n, m} T} \leq u_{n}\right)\right\}=0, \\
& \lim _{n \rightarrow+\infty}\left\{P\left(M_{k_{n-m}} \leq u_{n}\right)-P^{r^{-m} s_{n}}\left(M_{r_{n, m} T} \leq u_{n}\right)\right\}=0, \\
& \lim _{n \rightarrow+\infty}\left\{P\left(M_{k_{n}} \leq u_{n}\right)-P^{r^{m}}\left(M_{k_{n-m}} \leq u_{n}\right)\right\}=0, \\
& \lim _{n \rightarrow+\infty}\left\{P\left(M_{k_{n}} \leq u_{n}\right)-P^{m}\left(M_{\left[\frac{k_{n}}{m T}\right] T} \leq u_{n}\right)\right\}=0(m \neq 0) .
\end{aligned}
$$

The proof is omitted attending to its similarity with the proof of Lemma 4 in Temido and Canto e Castro (2003). However, we should remark that, due to the T-periodicity of $\left\{X_{n}\right\}$, we have, for instance,

$$
\begin{aligned}
P\left(\bigcap_{j=1}^{\beta_{n, m}}\left\{M\left(I_{j}^{*}\right) \geq u_{n}\right\}\right) & \leq \beta_{n, m} P\left(M\left(I_{1}^{*}\right) \geq u_{n}\right) \\
& \leq \beta_{n, m} \frac{\ell_{n}}{T} \sum_{j=1}^{T}\left(1-F_{j}\left(u_{n}\right)\right) \\
& =\beta_{n, m} \frac{\ell_{n}}{k_{n-m}} k_{n-m}\left(1-\bar{F}\left(u_{n}\right)\right)=o_{n}(1) .
\end{aligned}
$$

Now we may establish the following theorem which is the expected generalization of the Extremal Types Theorem concerned with the MSS class (Theorem 2.4). 
Theorem 3.1. Let $\left\{k_{n}\right\}$ be a nondecreasing integer sequence satisfying (3). Let $\left\{X_{n}\right\}$ be a T-periodic sequence and let $\left\{a_{n}>0\right\}$ and $\left\{b_{n}\right\}$ be real sequences such that lim $\sup k_{n}\left(1-\bar{F}\left(x / a_{n}+b_{n}\right)\right)<+\infty$ and $P\left(a_{n}\left(M_{k_{n}}-b_{n}\right) \leq x\right)$ converges to $G(x)$ for each continuity point of the nondegenerate d.f. $G$. If $\left\{X_{n}\right\}$ satisfies $D_{k_{n}}\left(x / a_{n}+b_{n}\right)$, for all $x$ in $\mathbb{R}$, then $G$ belongs to one of the three families of max-semistable laws.

Proof: Consider $u_{n}=x / a_{n}+b_{n}$. Let us first assume that (3) holds, with $r>1$. By the third limit of Lemma 3.1, we obtain

$$
\lim _{n \rightarrow+\infty} P\left(M_{k_{n-1}} \leq u_{n}\right)=G^{r^{-1}}(x) .
$$

Then, by Khintchine's Theorem, we have $G(x)=G^{r}(a x+b)$, where $a=\lim _{n \rightarrow+\infty} \frac{a_{n-1}}{a_{n}}$ and $b=\lim _{n \rightarrow+\infty} a_{n-1}\left(b_{n}-b_{n-1}\right)$. Thus, $G$ is max-semistable.

Moreover, if (3) holds with $r=1$, observe that for all positive integers $n$ and $m$ there is $p_{n}:=p_{n}(m)$ such that $\lim _{n \rightarrow+\infty} p_{n}=+\infty$ and $\frac{k_{n}}{m}=k_{p_{n}}(1+$ $\left.o_{n}(1)\right)$. Hence,

$$
\begin{aligned}
& \left|P\left(M_{k_{p_{n}}} \leq u_{n}\right)-P\left(M_{\left[\frac{k_{n}}{m T}\right] T} \leq u_{n}\right)\right| \\
& \quad \leq\left|\left[\frac{k_{n}}{m T}\right] T-k_{p_{n}}\right|\left(1-\frac{1}{T} \sum_{j=1}^{T}\left(1-F_{j}\left(u_{n}\right)\right)\right) \\
& \quad \rightarrow 0, n \rightarrow+\infty .
\end{aligned}
$$

Therefore, attending to the last limit in Lemma 3.1, we have

$$
\lim _{n \rightarrow+\infty} P\left(M_{k_{p_{n}}} \leq u_{n}\right)=G^{1 / m}(x) .
$$

Since $\lim _{n \rightarrow+\infty} P\left(M_{k_{p_{n}}} \leq u_{p_{n}}\right)=G(x)$, using again Khintchine's Theorem, we obtain the stability equation $G^{m}\left(a_{m} x+b_{m}\right)=G(x)$, which defines the class of max-stable laws.

Following Alpuim (1988), if $\left\{X_{n}\right\}$ is a T-periodic sequence of independent r.v.'s, then $\lim _{n \rightarrow+\infty} n\left(1-\bar{F}\left(u_{n}\right)=\tau\right.$ and $\lim _{n \rightarrow+\infty} P\left(M_{n} \leq u_{n}\right)=e^{-\tau}$ are 
equivalent. In what follows we deal with levels $u_{n}$ satisfying the above first limit, which will be denoted by $u_{n}\left(\tau, k_{n}\right)$. In this case we will say that $\left\{u_{n}\left(\tau, k_{n}\right)\right\}$ is a sequence of normalized levels for $\left\{X_{n}\right\}$ or for $\bar{F}$. If $\bar{F}$ is continuous, the existence of normalized levels, for any $\tau>0$, is granted if we take, for instance, $u_{n}=\bar{F}^{\longleftarrow}\left(1-\frac{\tau}{k_{n}}\right)$. However, for discrete $\bar{F}$, the existence of $u_{n}\left(\tau, k_{n}\right)$ deserves more attention. In fact, we can prove that, for some $\tau>0$, there exists $u_{n}\left(\tau, k_{n}\right)$ if and only if the sequence $\left\{x_{n}\right\}$ of discontinuity points of $\bar{F}$ satisfies $\lim _{n \rightarrow+\infty} \frac{1-\bar{F}\left(x_{n}\right)}{1-\bar{F}\left(x_{n-1}\right)}=r>1$. Consequently, if for some $\tau>0, u_{n}\left(\tau, k_{n}\right)$ exists, then for another $\tau^{\prime}>0$ the existence of levels $u_{n}\left(\tau^{\prime}, k_{n}\right)$ is equivalent to the existence of an integer $m$ such that $\tau=r^{m} \tau^{\prime}$. Thus, we need to define the set

$$
\Gamma\left(\bar{F}, k_{n}\right)=\left\{\tau>0: \exists\left\{u_{n}\right\}: \lim _{n \rightarrow+\infty} k_{n}\left(1-\bar{F}\left(u_{n}\right)\right)=\tau\right\} .
$$

We also observe that if $\bar{F}$ is a discrete d.f. and $\lim _{n \rightarrow+\infty} \frac{k_{n+1}}{k_{n}}=r>1$, then $\Gamma\left(\bar{F}, k_{n}\right)$ is not necessarily equal to $] 0,+\infty[$.

Generalizing a result of Leadbetter et al. (1983), Temido and Canto e Castro (2003) proved that if $\left\{k_{n}\right\}$ is an integer sequence satisfying (3) and the stationary sequence $\left\{X_{n}\right\}$ satisfies $D_{k_{n}}\left(u_{n}\left(\tau, k_{n}\right)\right)$, for all $\tau \in \Gamma\left(F, k_{n}\right)$, and $P\left(M_{k_{n}} \leq u_{n}\left(\tau, k_{n}\right)\right)$ converges, for some $\tau \in \Gamma\left(F, k_{n}\right)$, then, there is a real $\theta$ such that $\lim _{n \rightarrow+\infty} P\left(M_{k_{n}} \leq u_{n}\left(\tau, k_{n}\right)\right)=e^{-\theta \tau}$, for all such $\tau$.

Again, the parameter $\theta$ has been referred to as the extremal index of $\left\{X_{n}\right\}$. In the sequel we establish a similar result for T-periodic sequences. The proof is omitted attending to its similarity with the proof of Theorem 7 in Temido and Canto e Castro (2003).

Proposition 3.1. Let $\left\{X_{n}\right\}$ be a T-periodic sequence and $\left\{k_{n}\right\}$ an integer sequence satisfying (3). If $\left\{X_{n}\right\}$ satisfies $D_{k_{n}}\left(u_{n}\left(\tau, k_{n}\right)\right)$ for all $\tau \in$ $\Gamma\left(\bar{F}, k_{n}\right)$, and $P\left(M_{k_{n}} \leq u_{n}\left(\tau, k_{n}\right)\right)$ converges, for some $\tau \in \Gamma\left(\bar{F}, k_{n}\right)$, 
then there is a real $\theta$, such that $\lim _{n \rightarrow+\infty} P\left(M_{k_{n}} \leq u_{n}\left(\tau, k_{n}\right)\right)=e^{-\theta \tau}$, for all such $\tau$.

Once more we will say that $\left\{X_{n}\right\}$ has extremal index $\theta \in[0,1]$ if this theorem applies.

Ferreira and Martins (2003) introduce a family of local dependence conditions for T-periodic sequences satisfying Leadbetter's long range condition $D\left(u_{n}\right)$. In order to deal with max-semistable distributions, more precisely, with a number of $k_{n}$ r.v.'s of a T-periodic sequence, we will establish a simple adaptation of this family of conditions.

Definition 3.1. Let $\left\{k_{n}\right\}$ be a nondecreasing positive integer sequence and $\left\{u_{n}\right\}$ a real sequence. The sequence $\left\{X_{n}\right\}$ satisfies condition $D_{k_{n} T}^{(m)}\left(u_{n}\right)$, $m \geq 1$, if it satisfies $D_{k_{n}}\left(u_{n}\right)$ and if there are positive integer-valued sequences $\left\{s_{n}\right\}$ and $\left\{\ell_{n}\right\}$ satisfying $(6)$, such that

$$
\lim _{n \rightarrow \infty} k_{n} \frac{1}{T} \sum_{i=1}^{T} \sum_{j=i+m}^{r_{n}} P\left(X_{i}>u_{n} \geq M_{i+1, i+m-1}, X_{j}>u_{n}\right)=0,
$$

where $r_{n}:=r_{n, 0}$ and $M_{i+1, i}=-\infty$.

In order to establish a result that enables us to compute the extremal index we should remark that, using the arguments of Ferreira and Martins $(2003)$, we can prove that, if condition $D_{k_{n}, T}^{(m)}\left(u_{n}\right)$ holds for the Tperiodic sequence $\left\{X_{n}\right\}$, with $\left\{k_{n}\right\}$ a non-decreasing sequence of positive integers, then

$P\left(M_{k_{n}} \leq u_{n}\right)-\exp \left(\frac{k_{n}}{T} \sum_{i=1}^{T} P\left(X_{i}>u_{n} \geq M_{i+1, i+m-1}\right)\right) \rightarrow 0, n \rightarrow+\infty$.

Taking into account these results we can now compute the extremal index $\theta$ applying the expected generalization of the Corollary 2.1 of Ferreira and Martins (2003). 
Proposition 3.2. Let $\left\{k_{n}\right\}$ be a non-decreasing sequence of a positive integers and $\left\{X_{n}\right\}$ a T-periodic sequence satisfying $D_{k_{n}}\left(u_{n}\left(\tau, k_{n}\right)\right)$ for all $\tau$ in $\Gamma\left(\bar{F}, k_{n}\right)$ and $D_{k_{n}, T}^{(m)}\left(u_{n}\left(\tau_{0}, k_{n}\right)\right)$, for some positive integer $m$ and for some $\tau_{0}>0$. Thus $\left\{X_{n}\right\}$ has extremal index $\theta$ if and only if there exists

$$
\nu=\lim _{n \rightarrow \infty} k_{n} \frac{1}{T} \sum_{i=1}^{T} P\left(X_{i}>u_{n}\left(\tau_{0}, k_{n}\right) \geq M_{i+1, i+m-1}\right)
$$

and, in this case $\theta=\nu / \tau_{0}$.

\section{Applications}

4.1. The integer-valued MA model. We now consider a class of periodic integer-valued moving average models with exponential typetails. Let $\left\{Z_{n}\right\}$ be a sequence of T-periodic independent and integervalued r.v.'s with d.f. such that

$$
1-F_{Z_{j}}(x) \sim \zeta_{j}[x]^{\xi_{j}}\left(1+\lambda_{j}\right)^{-[x]}, x \longrightarrow+\infty,
$$

with $\xi_{j} \in \mathbb{R}, \zeta_{j}>0, \lambda_{j}>0$, for $j=1,2, \cdots, T$.

Define the T-periodic integer-valued sequence $\left\{X_{n}\right\}$ by

$$
X_{n}=\sum_{j=-\infty}^{+\infty} \beta_{j} \circ Z_{n-j}, \beta_{j} \in[0,1],
$$

where $\circ$ denotes binomial thinning. In order to guarantee almost sure convergence of the infinite series we take $\beta_{j}$ satisfying $\beta_{j}=O\left(|i|^{-\delta}\right)$ for some $\delta>2$ (Hall, 2003).

Hall and Scotto (2006) proved that there exist constants $A_{j}^{*}, \xi_{j}^{*}$ and $\lambda_{j}^{*}$ for which

$$
1-P\left(X_{j} \leq x\right) \sim A_{j}^{*}[x]^{\xi_{j}^{*}}\left(1+\lambda_{j}^{*}\right)^{-[x]}, x \in \mathbb{R},
$$

as long as $\xi_{j} \neq-1, j=1, \ldots T$. The expressions that define these constants are rather extensive and therefore we omit them here.

We now determine the limiting behavior of the maximum, $\widehat{M}_{k_{n}}$, of the associated independence sequence $\left\{\widehat{X}_{n}\right\}$ (with the same marginal 
distributions). We first observe that, taking a positive integer-valued sequence $\left\{b_{n}\right\}$ satisfying $b_{n} \rightarrow+\infty$ and such that $k_{n}^{(j)}=\left[\left(A_{j}^{*}\right)^{-1} b_{n}^{-\xi_{j}^{*}}(1+\right.$ $\left.\left.\lambda_{j}^{*}\right)^{b_{n}}\right]$ satisfies $(2)$, for each margin $X_{1}, \ldots, X_{T}$ we have,

$$
\begin{aligned}
k_{n}^{(j)} P\left(X_{j}>x+b_{n}\right) & \sim k_{n}^{(j)} A_{j}^{*}\left[x+b_{n}\right]_{j}^{\xi_{j}^{*}}\left(1+\lambda_{j}^{*}\right)^{-\left[x+b_{n}\right]} \\
& \sim k_{n}^{(j)} A_{j}^{*} \xi_{n}^{\xi_{j}^{*}}\left(1+\lambda_{j}^{*}\right)^{-[x]} \\
& \rightarrow\left(1+\lambda_{j}^{*}\right)^{-[x]}, x \rightarrow+\infty .
\end{aligned}
$$

Thus, for each fixed $j \in\{1,2, \ldots, T\}$, the distribution function of $X_{j}, F_{X_{j}}$, belongs to the domain of attraction of the discrete max-semistable d.f.

$$
G(x)=\exp \left(-\left(1+\lambda_{j}^{*}\right)^{-[x]}\right), x \in \mathbb{R} .
$$

However, for the T-periodic sequence $\left\{X_{n}\right\}$ we should work with the maximum of $k_{n}$ r.v.'s which implies that we can not consider T different values $k_{n}^{(1)}, \cdots, k_{n}^{(T)}$, but rather the same $k_{n}$ for all the T margins. Indeed we must find $\left\{k_{n}\right\}$ satisfying $(2)$ such that

$$
\begin{aligned}
k_{n}\left(1-\bar{F}\left(x+b_{n}\right)\right) & =k_{n}\left(1-\frac{1}{T} \sum_{j=1}^{T} F_{j}\left(x+b_{n}\right)\right) \\
& =\frac{1}{T} \sum_{j=1}^{T} k_{n}\left(1-F_{j}\left(x+b_{n}\right)\right) \longrightarrow \tau(x), n \longrightarrow+\infty .
\end{aligned}
$$

Define $\check{\lambda}=\min _{1 \leq j \leq T} \lambda_{j}^{*}$ and consider the set of indices $Q=\left\{q: \lambda_{q}^{*}=\right.$ $\check{\lambda}\} \subseteq\{1,2, \cdots, T\}$. Also, define $\check{\xi}=\max _{q \in Q} \xi_{q}^{*}$ and the set $M=\{m$ : $\left.\check{\xi}=\xi_{m}^{*}\right\} \subseteq Q$. Take $A^{*}=\sum_{m \in M} A_{m}^{*}$.

Theorem 4.1. Let $\left\{X_{n}\right\}$ be a T-periodic sequence defined by (7) with $\xi_{j} \neq-1, j=1, \ldots$ T. Then

$$
P\left(\widehat{M}_{k_{n}} \leq x+b_{n}\right) \longrightarrow \exp \left(-(1+\check{\lambda})^{-[x]}\right), x \in \mathbb{R},
$$


for some sequence $\left\{b_{n}\right\}$ of integers satisfying $\lim _{n \rightarrow+\infty} b_{n}=+\infty$ and such that $k_{n}=T\left[(1+\check{\lambda})^{b_{n}} b_{n}^{-\check{\xi}}\left(A^{*}\right)^{-1}\right]$ satisfies (2).

Proof: Indeed

$$
\begin{aligned}
& \sum_{j=1}^{T} A_{j}^{*}\left[x+b_{n}\right]^{\xi_{j}^{*}}\left(1+\lambda_{j}^{*}\right)^{-\left[x+b_{n}\right]}= \\
& =(1+\check{\lambda})^{-\left[x+b_{n}\right]}\left(\sum_{q \in Q} A_{q}^{*}\left[x+b_{n}\right]^{\xi_{q}^{*}}\right)+ \\
& +\sum_{j \in\{1, \cdots, T\} \backslash Q}\left(1+\lambda_{j}^{*}\right)^{-\left[x+b_{n}\right]} A_{j}^{*}\left[x+b_{n}\right]^{\xi_{j}^{*}} \\
& =(1+\check{\lambda})^{-\left[x+b_{n}\right]}\left(\left[x+b_{n}\right]^{\check{\xi}} \sum_{m \in M} A_{m}^{*}+\sum_{\ell \in Q \backslash M} A_{\ell}^{*}\left[x+b_{n}\right]^{\xi_{\ell}^{*}}\right)+ \\
& +\sum_{j \in\{1, \cdots, T\} \backslash Q}\left(1+\lambda_{j}^{*}\right)^{-\left[x+b_{n}\right]} A_{j}^{*}\left[x+b_{n}\right]^{\xi_{j}^{*}} \\
& =(1+\check{\lambda})^{-\left[x+b_{n}\right]}\left[x+b_{n}\right]^{\check{\xi}} A^{*} \times\left(1+\sum_{\ell \in Q \backslash M} A_{\ell}^{*}\left[x+b_{n}\right]^{\xi_{\ell}^{*}-\check{\xi}}+\right. \\
& \left.+\sum_{j \in\{1, \cdots, T\} \backslash Q}\left(\frac{1+\lambda_{j}^{*}}{1+\check{\lambda}}\right)^{-\left[x+b_{n}\right]} \frac{A_{j}^{*}}{A^{*}}\left[x+b_{n}\right]^{\xi_{j}^{*}-\check{\xi}}\right) \\
& =(1+\check{\lambda})^{-\left[x+b_{n}\right]}\left[x+b_{n}\right]^{\check{\xi}} A^{*}\left(1+o_{n}(1)\right) \\
& =(1+\check{\lambda})^{-[x]}(1+\check{\lambda})^{-b_{n}} b_{n}^{\check{\xi}} A^{*}\left(1+o_{n}(1)\right), n \rightarrow+\infty \text {. }
\end{aligned}
$$


Thus, taking $k_{n}=T\left[(1+\check{\lambda})^{b_{n}} b_{n}^{-\check{\xi}}\left(A^{*}\right)^{-1}\right]$, we obtain

$$
\begin{aligned}
k_{n}\left(1-\bar{F}\left(x+b_{n}\right)\right) & =k_{n}\left(\frac{1}{T} \sum_{j=1}^{T} A_{j}^{*}\left[x+b_{n}\right]^{\xi_{j}^{*}}\left(1+\lambda_{j}^{*}\right)^{-\left[x+b_{n}\right]}\right) \\
& \longrightarrow(1+\check{\lambda})^{-[x]}, x \in \mathbb{R},
\end{aligned}
$$

and, equivalently,

$$
P\left(\widehat{M}_{k_{n}} \leq x+b_{n}\right) \longrightarrow \exp \left(-(1+\check{\lambda})^{-[x]}\right), x \in \mathbb{R}
$$

We now prove that conditions $D_{k_{n}}\left(x+b_{n}\right)$ and $D_{k_{n}, T}^{(1)}\left(x+b_{n}\right)$ hold for $\left\{X_{n}\right\}$.

Like in Hall and Scotto (2006) and in Hall (2003) in the next results a further restriction on the coefficients $\beta_{i}$ is needed. Let

$$
w_{s}=\sharp\left\{j: \beta_{j T+s}=\max _{i} \beta_{i T+s}\right\}, s=1, \ldots, T .
$$

It will be required that $w_{s}=1$ for $s=1, \ldots, T$.

Theorem 4.2. Let $\left\{X_{n}\right\}$ be a T-periodic sequence defined by (7) with $w_{s}=1, s=1, \ldots, T$, and $\xi_{j} \neq-1, j=1, \ldots, T$.

For any sequence $\left\{b_{n}\right\}$ of integers satisfying $\lim _{n \rightarrow+\infty} b_{n}=+\infty$ such that $k_{n}=T\left[(1+\check{\lambda})^{b_{n}} b_{n}^{-\check{\xi}}\left(A^{*}\right)^{-1}\right]$ satisfies (2), conditions $D_{k_{n}}\left(x+b_{n}\right)$ and $D_{k_{n} T}^{(1)}\left(x+b_{n}\right)$ hold. 
Proof: Using the arguments of Hall and Temido (2005) we have, with $X_{i}^{*}=\left(X_{i_{1}}, \ldots, X_{i_{p}}\right)$ and $X_{j}^{*}=\left(X_{j_{1}}, \ldots, X_{j_{q}}\right)$ and for any $\varepsilon_{n}>0$,

$$
\begin{aligned}
& \left|P\left(X_{i}^{*} \leq u_{n}, X_{j}^{*} \leq u_{n}\right)-P\left(X_{i}^{*} \leq u_{n}\right) P\left(X_{j}^{*} \leq u_{n}\right)\right| \\
& \quad \leq \frac{k_{n}}{T} \sum_{j=1}^{T} P\left(x+b_{n} \leq X_{j} \leq x+b_{n}+2 \varepsilon_{n}\right) \\
& \quad+2 \frac{k_{n}}{T} \sum_{j=1}^{T} P\left(X_{j}-X_{j}^{\prime}>\varepsilon_{n}\right)+2 \frac{k_{n}}{T} \sum_{j=1}^{T} P\left(X_{j}-X_{j}^{\prime \prime}>\varepsilon_{n}\right)
\end{aligned}
$$

where $X_{j}^{\prime}=\sum_{i=-\infty}^{\ell_{n}-1} \beta_{i} \circ Z_{j-i}$ and $X_{j}^{\prime \prime}=\sum_{i=-\ell_{n}+1}^{\infty} \beta_{i} \circ Z_{j-i}$.

Since

$$
\begin{aligned}
P\left(X_{j}-X_{j}^{\prime}>\varepsilon_{n}\right) & =P\left(\sum_{i=\ell_{n}}^{+\infty} \beta_{i} \circ Z_{j-i}>\varepsilon_{n}\right) \\
& =P\left(\sum_{s=0}^{T-1} \sum_{i=0}^{+\infty} \beta_{\ell_{n}+i T+s} \circ Z_{j-\ell_{n}-i T-s}>\varepsilon_{n}\right) \\
& =P\left(\sum_{s=0}^{T-1} \sum_{i=\ell_{n} / T}^{+\infty} \beta_{i T+s} \circ Z_{j-i T-s}>\varepsilon_{n}\right)
\end{aligned}
$$

and similarly for $P\left(X_{j}-X_{j}^{\prime \prime}>\varepsilon_{n}\right)$, we deduce that

$$
\begin{aligned}
\left|P\left(X_{i}^{*} \leq u_{n}, X_{j}^{*} \leq u_{n}\right)-P\left(X_{i}^{*} \leq u_{n}\right) P\left(X_{j}^{*} \leq u_{n}\right)\right| \\
\quad \leq \frac{k_{n}}{T} \sum_{j=1}^{T}\left(1-F_{j}\left(x+b_{n}\right)-\left(1-F_{j}\left(x+b_{n}+2 \varepsilon_{n}\right)\right)\right) \\
\quad+2 \frac{k_{n}}{T} \sum_{j=1}^{T} P\left(\sum_{s=0}^{T-1} \sum_{i=\ell_{n} / T}^{+\infty} \beta_{i T+s} \circ Z_{j-i T-s}>\varepsilon_{n}\right) \\
\quad+2 \frac{k_{n}}{T} \sum_{j=1}^{T} P\left(\sum_{s=0}^{T-1} \sum_{i=-\infty}^{-\ell_{n} / T} \beta_{i T+s} \circ Z_{j-i T-s}>\varepsilon_{n}\right) .
\end{aligned}
$$


Attending that Hall and Scotto (2006) proved that

$$
E\left(\sum_{s=0}^{T-1} \sum_{i=-\infty}^{-\ell_{n} / T} \beta_{i T+s} \circ Z_{j-i T-s}\right)^{2}=O\left(\ell_{n}^{\delta-1}\right)
$$

using the Markov inequality and considering that $\left\{Z_{n}\right\}$ is T-periodic, we obtain

$$
\begin{aligned}
& \frac{k_{n}}{T} \sum_{j=1}^{T} P\left(\sum_{s=0}^{T-1} \sum_{i=\ell_{n} / T}^{+\infty} \beta_{i T+s} \circ Z_{j-i T-s}>\varepsilon_{n}\right) \\
& \leq \frac{k_{n}}{T} \sum_{j=1}^{T} E\left(\sum_{s=0}^{T-1} \sum_{i=\ell_{n} / T}^{+\infty} \beta_{i T+s} \circ Z_{j-i T-s}\right)^{2} / \varepsilon_{n}^{2} \\
& \leq C k_{n} \ell_{n}^{1-\delta} \varepsilon_{n}^{-2} .
\end{aligned}
$$

Considering for instance $\ell_{n}=\left[\frac{\left(k_{n}\right)^{\eta}}{T}\right] T$, with $1>\eta>\frac{\delta}{2(\delta-1)}$, and $\varepsilon_{n}^{2}=$ $k_{n}^{1-\delta / 2}$, the last term in (11) tends to zero when $n \rightarrow+\infty$. For the last term in (10) the procedure is the same.

Then, due to Khintchine's Theorem and (8), the first term of the right hand side of (10) goes to zero and thus $D_{k_{n}}\left(x+b_{n}\right)$ is satisfied for any integer-valued sequence $\left\{b_{n}\right\}$ under the assumptions of the theorem. Equivalently, $D_{k_{n}}\left(u_{n}\left(\tau, k_{n}\right)\right)$ holds for all $\tau \in \Gamma\left(\bar{F}, k_{n}\right)$.

Now in order to prove that $\left\{X_{n}\right\}$ satisfies condition $D_{k_{n}, T}^{(1)}\left(x+b_{n}\right)$, observe that, for $u_{n}=x+b_{n}$ and $\gamma_{n}<r_{n} \frac{T}{2}$, 


$$
\begin{aligned}
\frac{k_{n}}{T} \sum_{i=1}^{T} \sum_{j=i+1}^{r_{n} T} P\left(X_{i}>u_{n}, X_{j}>u_{n}\right) \\
\leq \frac{k_{n}}{T} \sum_{i=1}^{T}\left(\sum_{j=i+1}^{2 \gamma_{n}+T} P\left(X_{i}+X_{j}>2 u_{n}\right)\right. \\
\left.\quad+\sum_{j=2 \gamma_{n}+T+1}^{r_{n} T} P\left(X_{i}>u_{n}, X_{j}>u_{n}\right)\right) .
\end{aligned}
$$

Using Bernstein's inequality we get

$$
P\left(X_{i}+X_{j}>2 u_{n}\right) \leq E\left((1+h)^{X_{i}+X_{j}}\right)(1+h)^{-2 u_{n}}
$$

for $h>0$. Hall and Scotto (2006) proved that $E\left((1+h)^{X_{i}+X_{j}}\right)$ is uniformly bounded in $\mathrm{j}$, for $i=1,2, \ldots, T$, with $1<1+h<\sqrt{1+\check{\lambda} / \check{\beta}}$, where $\check{\beta}:=\max _{0 \leq s \leq T-1} \check{\beta}^{(s)}$ with $\check{\beta}^{(s)}=\max \left\{\beta_{1}^{(s)}, \beta_{2}^{(s)} / 2\right\}$ for $\beta_{1}^{(s)}=$ $\max _{j}\left\{\beta_{j T+s}: j \neq j^{\prime}\right\}<\beta^{(s)}\left(j^{\prime}\right.$ is such that $\left.\beta_{j^{\prime} T+s}=\max _{i} \beta_{i T+s}\right)$ and $\beta_{2}^{(s)}=\max _{t}\left\{\max _{j}\left\{\beta_{j T+s}+\beta_{j T+s+t}\right\}\right\}<2 \beta^{(s)}$.

Then

$$
\frac{k_{n}}{T} \sum_{i=1}^{T} \sum_{j=i+1}^{2 \gamma_{n}+T} P\left(X_{i}+X_{j}>2 u_{n}\right) \leq 3 k_{n} \gamma_{n} O\left((1+h)^{-2 b_{n}}\right) .
$$

Now, take $s_{n}=\left[k_{n}^{\alpha}\right]$ with $0<\alpha<1$ and $\eta+\alpha<1$ (in order to have $\frac{s_{n} \ell_{n}}{k_{n}} \rightarrow 0, n \rightarrow+\infty$ ) and $\alpha<\eta(\delta-1)-\delta / 2$ (in order to have $s_{n} \alpha_{n, \ell_{n}} \leq$ $\left.s_{n} k_{n} \ell_{n}^{1-\delta} \varepsilon_{n}^{-2} \rightarrow 0, n \rightarrow+\infty\right)$. Moreover, write $(1+h)^{2}:=(1+\check{\lambda})^{\vartheta}$, with $\vartheta>1$ such that $(1+\check{\lambda})^{\vartheta}<1+\check{\lambda} / \check{\beta}$, and choose $\gamma_{n}=(1+\check{\lambda})^{(\vartheta \mu-\varrho) b_{n}}$, with $\vartheta \mu-\varrho>0$ (to imply $\gamma_{n} \rightarrow+\infty$ ) and $1-\alpha>\vartheta \mu-\varrho$ (to imply $2 \gamma_{n}<T k_{n} / s_{n}$, for $n$ large). Then

$$
k_{n} \gamma_{n}(1+h)^{-2 b_{n}} \rightarrow 0, n \rightarrow+\infty,
$$


providing $\frac{1-\varrho}{1-\mu}<\vartheta$. For $\vartheta$ and $\alpha$ as above, consider $\mathcal{C}=\left\{(\mu, \varrho) \in \mathbb{R}^{2}\right.$ : $\left.\vartheta \mu-\varrho>0,1-\alpha>\vartheta \mu-\varrho, \frac{1-\varrho}{1-\mu}<\vartheta\right\}$ which is non-empty.

As far as the second sum in the right hand side of (12) is concerned, since the r.v.'s

$$
X_{j}^{\prime}=\sum_{l=-\infty}^{\gamma_{n}} \beta_{l} \circ Z_{j-l}=\sum_{s=0}^{T-1} \sum_{l=-\infty}^{\gamma_{n} / T} \beta_{l T+s} \circ Z_{j-(l T+s)}
$$

and $X_{i}^{\prime \prime}=\sum_{l=-\gamma_{n}}^{+\infty} \beta_{l} \circ Z_{i-l}=\sum_{s=0}^{T-1} \sum_{l=-\gamma_{n} / T}^{+\infty} \beta_{l T+s} \circ Z_{i-(l T+s)}$ are independent for $j-i>2 \gamma_{n}$ and using T-periodicity, we get, for $\varepsilon>0$,

$$
\begin{aligned}
\frac{k_{n}}{T} \sum_{i=1}^{T} \sum_{j=2 \gamma_{n}+T+1}^{r_{n} T} P\left(X_{i}>u_{n}, X_{j}>u_{n}\right) \\
\quad \leq \frac{k_{n}^{2}}{s_{n}} \sum_{i=1}^{T} P\left(X_{i}^{\prime}>u_{n}-\varepsilon\right) P\left(X_{i}^{\prime \prime}>u_{n}-\varepsilon\right) \\
+\frac{k_{n}^{2}}{s_{n}} \sum_{i=1}^{T} P\left(\sum_{s=0}^{T-1} \sum_{l=\gamma_{n} / T+1}^{+\infty} \beta_{l T+s} \circ Z_{i-(l T+s)}>\varepsilon\right) \\
+\frac{k_{n}^{2}}{s_{n}} \sum_{i=1}^{T} P\left(\sum_{s=0}^{T-1} \sum_{l=-\infty}^{-\gamma_{n} / T-1} \beta_{l T+s} \circ Z_{i-(l T+s)}>\varepsilon\right) .
\end{aligned}
$$

Taking notice that

$$
\begin{aligned}
P\left(X_{i}^{\prime}>u_{n}-\varepsilon\right) & =P\left(X_{i}^{\prime}>u_{n}-\varepsilon, X_{i}-X_{i}^{\prime}>-\varepsilon\right) \\
& \leq P\left(X_{i}^{\prime}>u_{n}-2 \varepsilon\right)=O\left(k_{n}^{-1}\right)
\end{aligned}
$$


and similarly for $P\left(X_{j}^{\prime \prime}>u_{n}-\varepsilon\right)$, we deduce that the first term of the right hand side of inequality (13) is negligible as $n \rightarrow+\infty$.

On the other hand, using the Bernstein's inequality and taking $\varepsilon>1$ and $h_{n}=\left(k_{n}^{2} / s_{n}\right)^{\theta / \epsilon}$ for some $\left.\theta \in\right] 1, \varepsilon[$, we obtain

$$
\begin{aligned}
& \frac{k_{n}^{2}}{s_{n}} \sum_{i=1}^{T} P\left(\sum_{s=0}^{T-1} \sum_{l=\gamma_{n} / T+1}^{+\infty} \beta_{l T+s} \circ Z_{i-(l T+s)}>\varepsilon\right) \\
& \leq \frac{k_{n}^{2}}{s_{n}} \sum_{i=1}^{T} E\left(\left(1+h_{n}\right)^{\sum_{s=0}^{T-1} \sum_{l=\gamma_{n} / T+1}^{+\infty} \beta_{l T+s} \circ Z_{i-(l T+s)}}\right) /\left(1+h_{n}\right)^{\varepsilon} .
\end{aligned}
$$

Following Hall (2003), we can prove that

$$
E\left(\left(1+h_{n}\right)^{\sum_{s=0}^{T-1} \sum_{l=\gamma n / T+1}^{+\infty} \beta_{l T+s} \circ Z_{i-(l T+s)}}\right)
$$

is bounded, uniformly in i, providing that $h_{n} / \gamma_{n} \rightarrow 0, n \rightarrow+\infty$. So, we choose $\theta$ such that $(2-\alpha) \theta / \varepsilon<\vartheta \mu-\varrho$ and also $\theta / \varepsilon<\frac{\vartheta-1}{2-\alpha}$ (to guarantee that the set $\mathcal{C} \cap\left\{(\mu, \varrho) \in \mathbb{R}^{2}:(2-\alpha) \theta / \varepsilon<\vartheta \mu-\varrho\right\}$ is non-empty). Then the left hand term of (14) does not exceed

$$
C \frac{k_{n}^{2}}{s_{n}} \frac{1}{\left(1+h_{n}\right) \epsilon} \leq C \frac{k_{n}^{2}}{s_{n}} \frac{1}{h_{n}^{\epsilon}}=C \frac{s_{n}^{\theta-1}}{k_{n}^{2(\theta-1)}} \rightarrow 0, n \rightarrow+\infty .
$$

We may now establish the limiting distribution of the maximum term, which turns out to be the same as if the sequence were independent.

Theorem 4.3. Let $\left\{X_{n}\right\}$ be a T-periodic sequence defined by (7), with $w_{s}=1, s=1, \ldots, T\left(w_{s}\right.$ defined by (9)), and $\xi_{j} \neq-1, j=1, \ldots T$. Considering $\left\{b_{n}\right\}$ a sequence of integers satisfying $\lim _{n \rightarrow+\infty} b_{n}=+\infty$ and such that $k_{n}=T\left[(1+\check{\lambda})^{b_{n}} b_{n}^{-\check{\xi}}\left(A^{*}\right)^{-1}\right]$ satisfies (2) then

$$
P\left(M_{k_{n}} \leq x+b_{n}\right) \longrightarrow \exp \left(-(1+\check{\lambda})^{-[x]}\right), x \in \mathbb{R} .
$$


Proof: The result follows by application of Theorem 3.2 and using the results of Theorems 4.1 and 4.2 .

4.2. First order max-autoregressive models. We now consider a first order max-autoregressive T-periodic model. Consider a r.v. $X_{0}$ and a T-periodic sequence $\left\{X_{n}\right\}_{n \geq 0}$ of non-negative integer-valued r.v.'s defined by

$$
X_{n}=\max \left\{X_{n-1}, Z_{n}\right\}-c_{n}, n \geq 1,
$$

where $c_{1}, \cdots, c_{T} \in \mathbb{N}, c_{n}=c_{n}+T$, for all $n \in \mathbb{Z}$, and $\left\{Z_{n}\right\}$ is an i.i.d. sequence of integer-valued r.v.'s with d.f. F satisfying (1). We assume that $\left\{Z_{n}\right\}$ is independent of $X_{0}$ and $H_{0}$ is defined by $H_{0}(x)=$ $\prod_{i=0}^{\infty} F\left(x+\sum_{l=0}^{i} c_{-l}\right), x \in \mathbb{R}$. Following Hall and Scotto (2006) we have

$H_{n}(x)=P\left(X_{n} \leq x\right)=\prod_{i=0}^{+\infty} F\left(x+\sum_{l=0}^{i} c_{n-l}\right)=\prod_{s=0}^{T-1} \prod_{j=0}^{+\infty} F\left(x+j S+S_{s, n}\right)$

where $S=\sum_{i=1}^{T} c_{i}$ and $S_{s, n}=\sum_{l=0}^{s} c_{n-l}$. We observe that $F(x)=$ $\frac{H_{n}\left(x-c_{n}\right)}{H_{n-1}(x)}, n \in \mathbb{N}$.

In order to establish the behavior of $M_{k_{n}}$ for some $k_{n}$ we present the following results.

Lemma 4.1. Consider the max-autoregressive integer-valued T-periodic sequence $\left\{X_{n}\right\}$ defined by (15). If $F$ satisfies (1) then the same holds with $H_{n}$, for all $n \in \mathbb{N}$, and there is an integer-valued sequence $\left\{b_{n}\right\}$ and a positive nondecreasing integer-valued sequence $\left\{k_{n}\right\}$ satisfying (2), such that

$$
\begin{array}{r}
\lim _{n \rightarrow+\infty} k_{n}\left(1-\frac{1}{T} \sum_{j=1}^{T} H_{j}\left(x+b_{n}\right)\right)=r^{-[x]} \frac{1}{T} \sum_{j=1}^{T} \gamma_{j}, \\
\text { with } \gamma_{j}=\lim _{n \rightarrow+\infty} \frac{1-H_{j}(x)}{1-F(x)}=\frac{\sum_{s=0}^{T-1} r^{-S_{s, j}}}{1-r^{-S}}, j=1, \cdots, T .
\end{array}
$$


Proof: Suppose that $\lim _{n \rightarrow+\infty} \frac{1-F(n)}{1-F(n+1)}=r>1$. Since $F$ is discrete this is equivalent to $\lim _{x \rightarrow+\infty} \frac{1-F(x)}{1-F(x+1)}=r>1$.

We have

$$
\begin{aligned}
\lim _{x \rightarrow+\infty} \frac{1-H_{j}(x)}{1-F(x)} & =\lim _{x \rightarrow+\infty} \frac{\sum_{s=0}^{T-1} \sum_{l=0}^{+\infty}\left(1-F\left(x+l S+S_{s, j}\right)\right)}{1-F(x)} \\
& =\lim _{x \rightarrow+\infty} \sum_{s=0}^{T-1} \sum_{l=0}^{+\infty} \frac{1-F\left(x+l S+S_{s, j}\right)}{1-F(x+l s)} \frac{1-F(s)}{1-F(x)} \\
& =\sum_{s=0}^{T-1} r^{-S_{s, j}} \sum_{l=0}^{+\infty} r^{-l S}=\frac{\sum_{s=0}^{T-1} r^{-S_{s, j}}}{1-r^{-S}}:=\gamma_{j}
\end{aligned}
$$

where we took into account the dominated convergence theorem. Thus $\frac{1-H_{j}(n)}{1-H_{j}(n+1)}=\frac{1-H_{j}(n)}{1-F(n)} \frac{1-F(n)}{1-F(n+1)} \frac{1-F(n+1)}{1-H_{j}(n+1)} \rightarrow r, n \rightarrow+\infty$.

However we can not apply Theorem 2.3 to $H_{j}$ because in this case we could obtain $\mathrm{T}$ different values $k_{n}^{(1)}, \cdots, k_{n}^{(T)}$ and the same for $b_{n}$. We apply this theorem firstly to the d.f. F. So, since F satisfies (1) we know that there exist a nondecreasing positive integer sequence $\left\{k_{n}\right\}$ satisfying (1) and an integer-valued sequence $\left\{b_{n}\right\}$ satisfying $\lim _{n \rightarrow+\infty} b_{n}=+\infty$ such that

$$
k_{n}\left(1-F\left(x+b_{n}\right)\right) \rightarrow r^{-[x]}, x \rightarrow+\infty .
$$

Since

$$
\begin{aligned}
k_{n}\left(1-H_{j}\left(x+b_{n}\right)\right) & =k_{n} \frac{1-H_{j}\left(x+b_{n}\right)}{1-F\left(x+b_{n}\right)}\left(1-F\left(x+b_{n}\right)\right) \\
& \rightarrow \gamma_{j} r^{-[x]}, n \rightarrow+\infty
\end{aligned}
$$


we get immediately

$$
\begin{aligned}
k_{n}\left(1-\bar{H}\left(x+b_{n}\right)\right) & =\frac{k_{n}}{T} \sum_{j=1}^{T}\left(1-H_{j}\left(x+b_{n}\right)\right) \\
& \rightarrow\left(\frac{1}{T} \sum_{j=1}^{T} \gamma_{j}\right) r^{-[x]}, n \rightarrow+\infty
\end{aligned}
$$

The limiting distribution of the maximum $M_{k_{n}}$ of the T-periodic sequence defined in (15) is obtained in the following result.

Theorem 4.4. Let $\left\{X_{n}\right\}$ be the T-periodic sequence defined by (15) with marginal d.f. F satisfying (1). Then

$$
\lim _{n \rightarrow+\infty} P\left(M_{k_{n}} \leq x+b_{n}\right)=\exp \left(-r^{-[x]} \frac{1}{T} \sum_{j=1}^{T} \gamma_{j}\left(1-r^{-c_{j+1}}\right)\right)
$$

for a suitable nondecreasing positive integer-valued sequence $\left\{k_{n}\right\}$ satisfying (2) and an integer-valued sequence $\left\{b_{n}\right\}$, with $b_{n} \rightarrow+\infty$, as $n \rightarrow+\infty$. The extremal index of the sequence is given by

$$
\theta=\sum_{j=1}^{T} \gamma_{j}\left(1-r^{-c_{j+1}}\right) / \sum_{j=1}^{T} \gamma_{j}
$$

Proof: Following the proof of last theorem we know that there are $\left\{k_{n}\right\}$ and $\left\{b_{n}\right\}$ such that $k_{n} b\left(1-H_{j}\left(x+b_{n}\right)\right) \rightarrow r^{-[x]}, n \rightarrow+\infty$, for all $j$ in $\{1,2, \cdots, T\}$. Thus replacing $1-H_{j}\left(x+b_{n}\right)=O\left(\frac{1}{n}\right)$ by $1-H_{j}\left(x+b_{n}\right)=$ $O\left(\frac{1}{k_{n}}\right)$, for all $j$, in the proof of the theorem 4.1 of Hall and Scotto (2006) we obtain that $D_{k_{n}}\left(x+b_{n}\right)$ and $D_{k_{n} T}^{(2)}\left(x+b_{n}\right)$ hold. The extremal index $\theta$ is given by 


$$
\begin{aligned}
\theta & =\lim _{n \rightarrow+\infty} \frac{\sum_{j=1}^{T} P\left(X_{j}>u_{n} \geq X_{j+1}\right)}{\sum_{j=1}^{T} P\left(X_{j}>u_{n}\right)} \\
& =\frac{\lim _{n \rightarrow+\infty} \sum_{j=1}^{T} \frac{1-H_{j}\left(x+b_{n}\right)}{1-F\left(x+b_{n}\right)}\left(1-\frac{1-H_{j}\left(x+b_{n}+c_{j+1}\right)}{1-H_{j}\left(x+b_{n}\right)}\right)}{\lim _{n \rightarrow+\infty} \sum_{j=1}^{T} \frac{1-H_{j}\left(x+b_{n}\right)}{1-F\left(x+b_{n}\right)}} \\
& =\sum_{j=1}^{T} \gamma_{j}\left(1-r^{-c_{j+1}}\right) / \sum_{j=1}^{T} \gamma_{j} .
\end{aligned}
$$

Due to the last lemma, we get

$$
\lim _{n \rightarrow+\infty} P\left(\hat{M}_{k_{n}} \leq x+b_{n}\right)=\exp \left(-r^{-[x]} \frac{1}{T} \sum_{j=1}^{T} \gamma_{j}\right)
$$

and then we deduce (16).

4.3. Second order max-autoregressive models. Consider two r.v.'s $X_{0}$ and $X_{1}$ and a T-periodic integer-valued second order max-autoregressive sequence $\left\{X_{n}\right\}$ defined by

$$
X_{n}=\max \left\{X_{n-2}, Z_{n}\right\}-c_{n}, n \geq 2,
$$

where $c_{1}, \cdots, c_{T} \in \mathbb{N}, c_{n}=c_{n}+T$, for all $n \in \mathbb{Z}$, and $\left\{Z_{n}\right\}$ is an i.i.d. sequence of integer-valued r.v.'s with d.f. F satisfying (1). We assume that $\left\{Z_{n}\right\}, X_{0}$ and $X_{1}$ are independent. 
In this case we have

$$
\begin{aligned}
H_{n}(x) & =\prod_{j=0}^{+\infty} F\left(x+\sum_{l=0}^{j} c_{n-2 l}\right) \\
& =\prod_{s=0}^{T-1} \prod_{j=0}^{+\infty} F\left(x+j \sum_{k=0}^{T-1} c_{n-2 k}+\sum_{k=0}^{s} c_{n-2 k}\right),
\end{aligned}
$$

where $H_{0}$ and $H_{1}$ are assumed to be defined by

$$
H_{0}(x)=\prod_{j=0}^{+\infty} F\left(x+\sum_{l=0}^{j} c_{-2 l}\right)
$$

and

$$
H_{1}(x)=\prod_{j=0}^{+\infty} F\left(x+\sum_{l=0}^{j} c_{1-2 l}\right) .
$$

Using the periodicity of the constants $c_{n}, n \in \mathbb{Z}$, we obtain

$$
S_{n}^{*}:=\sum_{k=0}^{T-1} c_{n-2 k}= \begin{cases}\sum_{k=0}^{T-1} c_{k} & \text { Todd } \\ 2 \sum_{\substack{k=1 \\ T / 2}}^{T / 2} c_{2 k-2} & T \text { even, } n \text { even } . \\ 2 \sum_{k=1}^{T} c_{2 k-1} & T \text { even, } n \text { odd }\end{cases}
$$

Thus $H_{n}(x)=\prod_{s=0}^{T-1} \prod_{j=0}^{+\infty} F\left(x+j S_{n}^{*}+S_{n, s}^{*}\right)$ with $S_{n, s}^{*}=\sum_{k=0}^{s} c_{n-2 k}$.

As in the previous section we obtain

$$
\lim _{x \rightarrow+\infty} \frac{1-H_{j}(x)}{1-F(x)}=\frac{\sum_{s=0}^{T-1} r^{-S_{s, j}^{*}}}{1-r^{-S_{j}^{*}}}:=\beta_{j}
$$


and then

$$
k_{n}\left(1-\bar{H}\left(x+b_{n}\right)\right)=k_{n}\left(1-\frac{1}{T} \sum_{j=1}^{T} H_{j}\left(x+b_{n}\right)\right) \rightarrow r^{-[x]} \frac{1}{T} \sum_{j=1}^{T} \beta_{j}
$$

We now state our last result.

Theorem 4.5. Let $\left\{X_{n}\right\}$ be the T-periodic sequence defined by (17) with marginal d.f. F satisfying (1). Then

$$
\lim _{n \rightarrow+\infty} P\left(M_{k_{n}} \leq x+b_{n}\right)=\exp \left(-r^{-[x]} \frac{1}{T} \sum_{j=1}^{T} \beta_{j}\left(1-r^{-c_{j+2}}\right)\right)
$$

for a suitable nondecreasing positive integer-valued sequence $\left\{k_{n}\right\}$ satisfying (2) and an integer-valued sequence $\left\{b_{n}\right\}$, with $b_{n} \rightarrow+\infty$, as $n \rightarrow+\infty$. The extremal index of the sequence is given by

$$
\theta=\sum_{j=1}^{T} \beta_{j}\left(1-r^{-c_{j+2}}\right) / \sum_{j=1}^{T} \beta_{j}
$$

Proof: Using similar arguments to those in Hall and Scotto (2006) for the first order max-autoregressive periodic model, we can prove that condition $D\left(u_{n}\right)$ holds for $\left\{X_{n}\right\}$. Then taking $\ell_{n}=o\left(k_{n}\right)$, the condition $D_{k_{n}}\left(u_{n}\right)$ holds as well. We now prove that condition $D_{k_{n} T}^{(3)}\left(u_{n}\right)$ is satisfied. Note that, unlike the first order max-autoregressive model of the previous section, condition $D_{k_{n} T}^{(2)}\left(u_{n}\right)$ does not hold. For this type of sequences, the clusters of exceedances are formed by alternating observations whereas in the previous section clusters were formed by consecutive observations. 
Due to

$$
\begin{aligned}
& \frac{k_{n}}{T} \sum_{i=1}^{T} \sum_{j=i+3}^{r_{n} T} P\left(X_{i}>u_{n}, X_{i+1} \leq u_{n}, X_{i+2} \leq u_{n}, X_{j}>u_{n}\right) \\
& =\frac{k_{n}}{T} \sum_{i=1}^{T} \sum_{l=3}^{r_{n} T-i} P\left(X_{i}>u_{n}, X_{i+1} \leq u_{n}, X_{i+2} \leq u_{n}, X_{i+l}>u_{n}\right) \\
& \leq \frac{k_{n}}{T} \sum_{i=1}^{T} \sum_{\substack{l=4 \\
l \text { even }}}^{r_{n} T} P\left(X_{i}>u_{n}, X_{i+2} \leq u_{n}, X_{i+l}>u_{n}\right) P\left(X_{i+1} \leq u_{n}\right) \\
& \quad+\frac{k_{n}}{T} \sum_{i=1}^{T} \sum_{\substack{l=3 \\
l \text { lodd }}}^{r_{n} T} P\left(X_{i}>u_{n}, X_{i+2} \leq u_{n}\right) P\left(X_{i+1} \leq u_{n}, X_{i+l}>u_{n}\right)
\end{aligned}
$$

and, for $l$ even, we get

$$
\begin{aligned}
& P\left(X_{i}>u_{n}, X_{i+2} \leq u_{n}, X_{i+l}>u_{n}\right) \\
& \quad=P\left(X_{i}>u_{n}, X_{i+2} \leq u_{n}\right)\left(1-\prod_{j=0}^{\frac{l-4}{2}} F\left(u_{n}+\sum_{m=0}^{j} c_{i+l-2 m}\right)\right) \\
& \quad \leq P\left(X_{i}>u_{n}\right)\left(1-\prod_{j=0}^{+\infty} F\left(u_{n}+\sum_{m=0}^{j} c_{i+l-2 m}\right)\right) \\
& \quad \leq\left(1-H_{i}\left(u_{n}\right)\right)\left(1-H_{i+l}\left(u_{n}\right)\right)
\end{aligned}
$$


we obtain

$$
\begin{aligned}
& \frac{k_{n}}{T} \sum_{i=1}^{T} \sum_{j=i+3}^{r_{n} T} P\left(X_{i}>u_{n}, X_{i+1} \leq u_{n}, X_{i+2} \leq u_{n}, X_{j}>u_{n}\right) \\
& \leq \frac{k_{n}}{T} \sum_{i=1}^{T} \sum_{j=i+3}^{r_{n} T}\left(1-H_{i}\left(u_{n}\right)\right)\left(1-H_{i+l}\left(u_{n}\right)\right) \\
& \leq k_{n} r_{n} O\left(\frac{1}{k_{n}}\right) O\left(\frac{1}{k_{n}}\right) \rightarrow 0, n \rightarrow+\infty
\end{aligned}
$$

Applying Theorem 3.2 the extremal index is given by

$$
\begin{aligned}
\theta & =\lim _{n \rightarrow+\infty} \frac{\sum_{j=1}^{T} P\left(X_{j}>u_{n} \geq X_{j+2}\right) P\left(X_{j+1} \leq u_{n}\right)}{\sum_{j=1}^{T} P\left(X_{j}>u_{n}\right)} \\
& =\frac{\lim _{n \rightarrow+\infty} \sum_{j=1}^{T} \frac{1-H_{j}\left(u_{n}\right)}{1-F\left(u_{n}\right)}\left(1-\frac{1-H_{j}\left(u_{n}+c_{j+2}\right)}{1-H_{j}\left(u_{n}\right)}\right) H_{j+1}\left(u_{n}\right)}{\lim _{n \rightarrow+\infty} \sum_{j=1}^{T} \frac{1-H_{j}\left(u_{n}\right)}{1-F\left(u_{n}\right)}} \\
& =\sum_{j=1}^{T} \beta_{j}\left(1-r^{-c_{j+2}}\right) / \sum_{j=1}^{T} \beta_{j} .
\end{aligned}
$$

\section{References}

Al-Osh, M. A. and Alzaid, A. A., First order integer-valued autoregressive INAR(1) process, J. Time Series Anal, 8, 261-275, (1987).

Al-Osh, M. A. and Alzaid, A. A., Integer-valued moving average (INMA) process, Statistical Papers, 29, 281-300, (1988). 
Alpuim, M.T. (1988) Contribuições à teoria de extremos em sucessões dependentes, Ph.D. Thesis. University of Lisbon.

Alzaid, A. A. and Al-Osh, M. A. (1988) First-order integer-valued autoregressive (INAR(1)) process: distributional and regression properties, Statist. Neerl. 42, 53-61.

Alzaid, A. A. and Al-Osh, M. A. (1990) An integer-valued pth-order autoregressive structure $(\operatorname{INAR}(\mathrm{p}))$ process, J. Appl. Probab. 27, $314-324$.

Alzaid, A. A. and Al-Osh, M. A. (1993) Some autoregressive moving average processes with generalized Poisson marginal distributions, Ann. Inst. Statist. Math. 45, 223-232.

Anderson, C.W. (1970). Extreme value theory for a class of discrete distribution with applications to some stochastic processes. J. Appl. Prob. 7, p. 99-113.

Brännäs, K. and Hall, A. (2001), Estimation in integer-valued moving average models, Applied Stochastic Processes in Business and Industry 17 (3), 277-291.

Cameron, A. C. and Trivedi, P. (1998) Regression Analysis of Count Data, Cambridge University Press, Oxford.

Canto e Castro, L., de Haan, L. and Temido, M.G. (2002) Rarely observed sample maxima. Theory Probab. Appl. 454, 779-782.

Chernick, M., Hsing, T. and McCormick, W. (1991), Calculating the extremal index for a class of stationary sequences. Adv. Appl. Prob. 6, 711-731.

Ferreira, H. and Martins, A.P. (2003). The extremal index of subsampled sequences with strong local condition. Revstat. 1, 16-24.

Grinevich, I.V. (1992) Max-semistable limit laws under linear and power normalizations. Theory Probab. Appl. 38, 640-650. 
Grinevich, I.V. (1993) Domains of attraction of the max-semistable laws under linear and power normalizations. Theory Probab. Appl. 38, 640-650.

Hall, A. (1996) Maximum term of a particular sequence with discrete margins. Comm. Stat. - Theory and Methods 28, 721-736.

Hall, A. (2001), Extremes of integer-valued moving averages models with regularly varying tails. Extremes 4, 219-239.

Hall, A. (2003), Extremes of integer-valued moving averages models with exponential type tails. Extremes 6, 361-379.

Hall, A. and Temido, M.G. (2007), On the maximum term of MA and Max-AR models with margins in Anderson's class, Theory Probab. Appl. 51, 291-304.

Hall, A. and Scotto, M. (2006) Extremes of periodic integer-valued sequences with exponential type tails, Revstat - Statistical Journal 4, 249-273.

Kedem, B. and Fokianos, K (2002) Regression models for time series analysis, John Wiley and Sons.

Leadbetter, M. R., Lindgren, G. and Rootzén, H. (1983), Extremes and Related Properties of Random Sequences and Processes (SpringerVerlag, Berlin).

McCormick, W. and Park, Y. (1992) Asymptotic analysis of extremes from autoregressive negative binomial processes. Journal of Applied Probability 29, 904-920.

MacDonald, I.L. and Zuchini, W. (1997) Hidden Markov and other models for discrete-valued time series. Chapman and Hall.

McKenzie, E. (1985) Some simple models for discrete variate time series, Water Res. Bull. 21, 645-650. 
McKenzie, E. (1986) Auto regressive-moving-average processes with negative binomial and geometric marginal distribution. Adv. in Appl. Probab. 18, 679-705.

McKenzie, E. (1988) Some ARMA models for dependent sequences of Poisson counts, Adv. Appl. Probab. 20, 822-835.

McKenzie, E. (2003) Discrete Variate Time Series, in Handbook of Statistics 21, 573-606, (Eds. D.N. Shanbhag and C.R. Rao) Elsevier Science Pub Co.

Pancheva, E. (1992) Multivariate max-semistable distributions, Theory Probab. Appl., 18, 679-705.

Temido, M.G. (2000). Classes de leis limite em teoria de valores extremosestabilidade e semiestabilidade. Ph.D. Thesis. University of Coimbra.

Temido, M.G. (2002) Domínios de atracção de funções de distribuição discretas, Novos Rumos em Estatística - Proceedings of "IX Congresso da Sociedade Portuguesa de Estatística", Edition of the Portuguese Society of Statistics .

Temido, M.G. and Canto e Castro, L. (2003) Max-semistable laws in extremes of stationary random sequences, Theory Probab. Appl., 47, $365-374$.

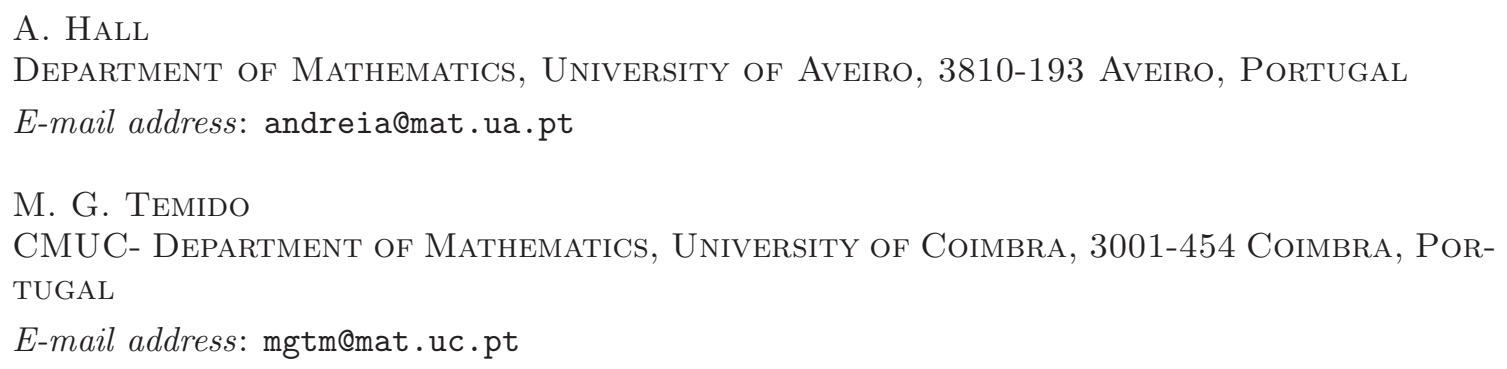

